\section{Case Reports in Ophthalmology}

\title{
The Effect of a Diving Mask on Intraocular Pressure in a Healthy Population
}

\author{
Catherina Josephine Goenadi ${ }^{a}$ David Zhiwei Law ${ }^{b, c}$ Jia Wen Lee \\ Ee Lin Ong ${ }^{a} \quad$ Wai Kitt Chee ${ }^{a}$ Jason Cheng ${ }^{a}$ \\ ${ }^{a}$ Ophthalmology and Visual Sciences Department, Khoo Teck Puat Hospital, \\ Singapore, Singapore; ${ }^{b}$ National Healthcare Group Eye Institute, Tan Tock Seng Hospital, \\ Singapore, Singapore; ${ }^{c}$ Vision Performance Centre, Military Medicine Institute, Singapore \\ Armed Forces, Singapore, Singapore
}

\section{Keywords}

Diving mask · Swimming goggles $\cdot$ Intraocular pressure $\cdot$ Glaucoma

\begin{abstract}
Purpose: Swimming goggles increase the intraocular pressure (IOP) via the periorbital frame pressure and suction effect. In comparison, diving masks have a larger frame rim and incorporate the nose. The exact effect(s) of diving masks on IOP is unknown. This study evaluates the influence of diving masks on IOP in normal, healthy subjects. Methods: Tonometry was performed in both eyes of all subjects with an AVIA ${ }^{\circledR}$ Tono-Pen by a single investigator. Measurements were taken at baseline without the diving mask and with the subjects wearing a small-volume, double-window diving mask, but with the mask lenses removed. Two IOP readings in each eye were measured, and an additional reading was measured if the difference between the initial 2 was $\geq 2 \mathrm{~mm} \mathrm{Hg}$. Central corneal thickness (CCT) was also measured in each eye, using a contact pachymeter (OcuScan ${ }^{\circledR}$ Alcon). Results: Forty eyes of 20 healthy volunteers (age $29.7 \pm 9.3$ years; range $21-52$ ) were included. The mean CCT was $544.4 \pm$ $43.5 \mu \mathrm{m}$. The mean IOP before the diving mask was worn had been $17.23 \pm 2.18 \mathrm{~mm} \mathrm{Hg} \mathrm{(} \mathrm{n}=$ 40). The IOP decreased by $0.43 \mathrm{~mm} \mathrm{Hg}(p<0.05)$ to $16.80 \pm 2.57 \mathrm{~mm} \mathrm{Hg}$ after the diving mask had been put on. There was no correlation between IOP change and age ( $r=0.143, p=$ $0.337)$, gender $(r=-0.174, p=0.283)$ or CCT $(r=-0.123, p=0.445)$. Conclusion: There was
\end{abstract}


no increase in IOP after the diving mask had been worn. A small but statistically significant decrease in IOP was observed. This study demonstrates that unlike swimming goggles, the strap tension and frame pressure on the periorbital tissue from a diving mask does not increase IOP. Diving masks may be a suitable alternative to swimming goggles for patients with advanced glaucoma or glaucoma filtration surgery.

(C) 2016 The Author(s)

Published by S. Karger AG, Basel

\section{Introduction}

Glaucoma is the leading cause of irreversible blindness worldwide according to the World Health Organization [1]. An elevated intraocular pressure (IOP) is widely regarded as a significant risk factor for glaucoma development and progression [2, 3]. Recreational scuba diving is an increasingly popular sport, and the professional association of diving instructors (PADI) has issued over 14 million diver certifications in the last 16 years [4].

Diving can affect the IOP in a number of ways; firstly from the change in the internal mask air pressure at different underwater depths, secondly from the inhaling or exhaling from the nose, and thirdly through the diving mask itself. The first and second factors can be controlled by the diver by exhaling from the nose during descent, also known as equalizing the mask pressure. Senn et al. [5] found that diving mask pressure spikes were less common in experienced divers compared to beginners. Failing to equalize the diving mask pressure can lead to ocular barotrauma, which ranges from lid edema, ecchymosis and subconjunctival hemorrhage to more serious injuries such as hyphema and subperiosteal orbital hemorrhage [6, 7]. The diving mask itself may also affect the IOP. Swimming goggles are known to increase the IOP, with larger-frame goggles having less effect than smaller-frame ones [8-10].

To our knowledge, the effect of diving masks on IOP is still currently unknown. Our study evaluates the influence of diving masks on IOP in normal healthy subjects.

\section{Materials and Methods}

We recruited 20 normal healthy subjects with no existing ocular condition.

Tonometry was performed in both eyes of all subjects with an AVIA ${ }^{\circledR}$ Tono-Pen (Reichert Technologies, Depew, N.Y., USA) by a single investigator. Measurements were taken at baseline without the diving mask and with the subjects wearing a small-volume, double-window diving mask, but with the mask lenses removed (fig. 1). One drop of tetracaine was instilled in both eyes 2 min prior to the first reading. IOP was measured in a sitting position with the subject in primary gaze, and the participants were allowed to blink between the individual measurements. Two IOP readings in each eye were measured, and an additional reading was measured if the difference between the initial 2 was $\geq 2 \mathrm{~mm} \mathrm{Hg}$. We obtained the mean of these readings. Central corneal thickness (CCT) was also measured in each eye using a contact pachymeter (OcuScan ${ }^{\circledR}$ Alcon; Alcon, Fort Worth, Tex., USA).

Statistical analysis was performed using SPSS 22.0 software (SPSS Inc., Chicago, Ill., USA). IOP measurements from both eyes were used in this study, and the Shapiro-Wilk test was first used to demonstrate that the data were normally distributed. Differences between IOP measurements with and without the diving mask were evaluated using the paired t test. Pearson correlation coefficients were used to analyze the relationship between IOP change and age, gender or CCT. We considered a $p$ value $<0.05$ statistically significant. 


\section{Results}

Twenty subjects, 12 females and 8 males, with a mean age of $29.7 \pm 9.3$ years were recruited for this study. As both eyes of each study subject were considered, we analyzed a total of 40 eyes. The mean CCT was $544.4 \pm 43.5 \mu \mathrm{m}$.

The mean IOP before the diving mask was worn was $17.23 \pm 2.18 \mathrm{~mm} \mathrm{Hg}(\mathrm{n}=40)$. The IOP decreased by $0.43 \mathrm{~mm} \mathrm{Hg}(\mathrm{p}<0.05)$ to $16.80 \pm 2.57 \mathrm{~mm} \mathrm{Hg}$ after the diving mask had been put on. The scatter plot of IOP measurements before and after donning of the diving mask is illustrated (fig. 2). There was no correlation between IOP change and age ( $\mathrm{r}=0.143$, $p=0.337)$, gender $(r=-0.174, p=0.283)$ or CCT $(r=-0.123, p=0.445)$.

\section{Discussion}

The effect of donning of a diving mask on IOP and hence its significance to divers with glaucoma is not well addressed in the ophthalmic literature. Our study, although not performed while diving, shows that there was no increase in IOP after the diving mask had been worn. Although we found a $0.43 \mathrm{~mm} \mathrm{Hg}$ decrease in IOP when the diving mask was worn, this value was within the margin of error of Tono-Pen AVIA ${ }^{\circledR}$ [11], and thus this decrease of IOP, although statistically significant in our study, was not clinically significant.

Swimming goggles are known to increase the IOP, and Morgan et al. [8] concluded that smaller swimming goggle frames had a greater effect on IOP than larger frames. They postulated that the headband tension of the swimming goggles transmitted into the orbit through the rubber seal increases orbital tissue pressure, compressing the globe and leading to the sustained IOP rise observed. This theory is consistent with our findings, as a larger diving mask frame that sits on the bony orbit will exert less pressure on the periocular soft tissue and have less effect on the IOP. Another difference between swimming goggles and a diving mask is that the nose is also enclosed in the latter. Inhaling and exhaling through the nose changes the internal pressure of the mask; therefore, the suction effect seen in swimming goggles would not apply to a diving mask.

Although a different mask design could have a different effect on IOP, the diving mask we chose was that of a small-volume design. As such, we may extrapolate our finding to other diving masks of intermediate- or large-volume designs, as these would exert less pressure on the periorbital tissue due to their larger size frames, and hence wearing them would probably not result in any increase in IOP. Based on the finding from our study, we would therefore propose that a diving mask may be a suitable alternative to swimming goggles for patients with advanced glaucoma or glaucoma filtration surgery.

This study simulated only partial effects of the scuba diving mask because we removed the lenses to allow IOP measurement that resulted in the loss of an airtight seal and eliminated the usual suction effect. This study also did not look at the effects of the change in underwater mask air pressure at different water depths. Although Senn et al. [5] found that pressure equalization of a regular mask would prevent large IOP spikes, the pressure difference between the inside and outside of the mask still fluctuated by $19 \mathrm{~mm} \mathrm{Hg}$, parallel to respiration. It is not known how different diving mask designs may affect the fluctuations of pressure difference between the inside and outside of the diving masks and hence what long-term effect this can have on ocular tissues.

Future studies could potentially be carried out in a hyperbaric chamber to simulate the underwater effects. In addition, a longitudinal study looking at the effects of regular diving in 
healthy subjects and glaucoma patients may help determine long-term impacts of these pressure fluctuations on the optic nerve.

\section{Statement of Ethics}

Informed consent was obtained from all subjects. The study was approved by National Healthcare Group Domain Specific Review Board and was performed in accordance with the Declaration of Helsinki.

\section{Disclosure Statement}

This study was supported by a grant from The Alexandra Health Enabling Grant, Alexandra Health, Singapore. All the authors report no financial disclosures.

\section{References}

1 Quigley HA, Broman AT: The number of people with glaucoma worldwide in 2010 and 2020. Br J Ophthalmol 2006;90:262-267.

-2 Sommer A: Intraocular pressure and glaucoma. Am J Ophthalmol 1989;107:186-188

-3 Leske MC, Heijl A, Hyman L, et al: Factors for progression and glaucoma treatment: the Early Manifest Glaucoma Trial. Curr Opin Ophthalmol 2004;15:102-106.

4 PADI Statistics: https://www.padi.com/scuba-diving/about-padi/statistics/.

-5 Senn P, Helfenstein U, Senn ML, Schmid MK, Schipper I: Ocular barostress and barotrauma. A study of 15 scuba divers (in German). Klin Monbl Augenheilkd 2001;218:232-236; discussion 237-238.

-6 Gómez-Ledesma I, Mencía-Gutíerrez E, Gutiérrez-Díaz E, Alonso-Santiago MA: Orbital subperiosteal hemorrhage while scuba diving. Orbit 2006;25:19-22.

7 Rudge FW: Ocular barotrauma caused by mask squeeze during a scuba dive. South Med J 1994;87:749750 .

8 Morgan WH, Cunneen TS, Balaratnasingam C, Yu DY: Wearing swimming goggles can elevate intraocular pressure. Br J Ophthalmol 2008;92:1218-1221.

-9 Starr CE, Radcliffe NM: Swimming goggles and elevated intraocular pressure. Br J Ophthalmol 2009;93:700.

10 Kang MH, Morgan WH, Balaratnasingam C, Anastas C, Yu DY: Case of normal tension glaucoma induced or exacerbated by wearing swimming goggles. Clin Experiment Ophthalmol 2010;38:428-429.

-11 Schweier C, Hanson JV, Funk J, Töteberg-Harms M: Repeatability of intraocular pressure measurements with Icare PRO rebound, Tono-Pen AVIA, and Goldmann tonometers in sitting and reclining positions. BMC Ophthalmol 2013;13:44. 


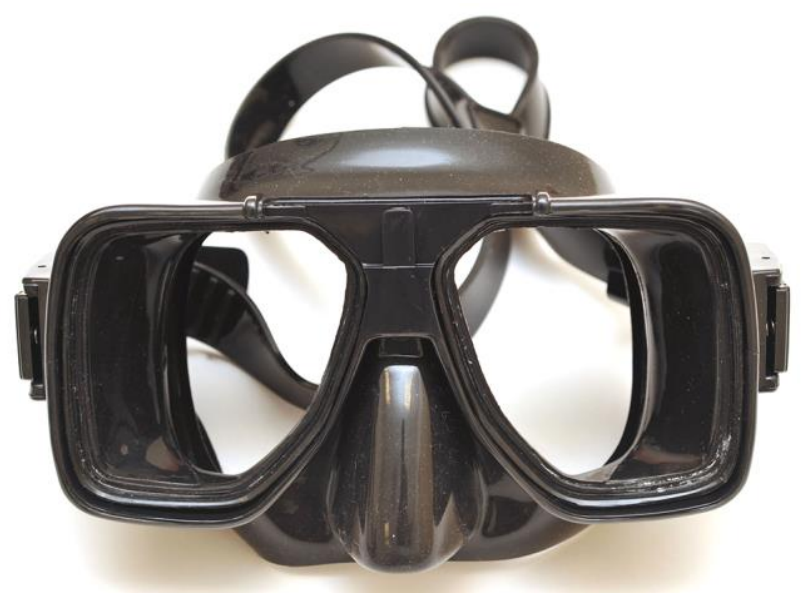

Fig. 1. Photograph of the dive mask used in our study with the mask lenses removed.

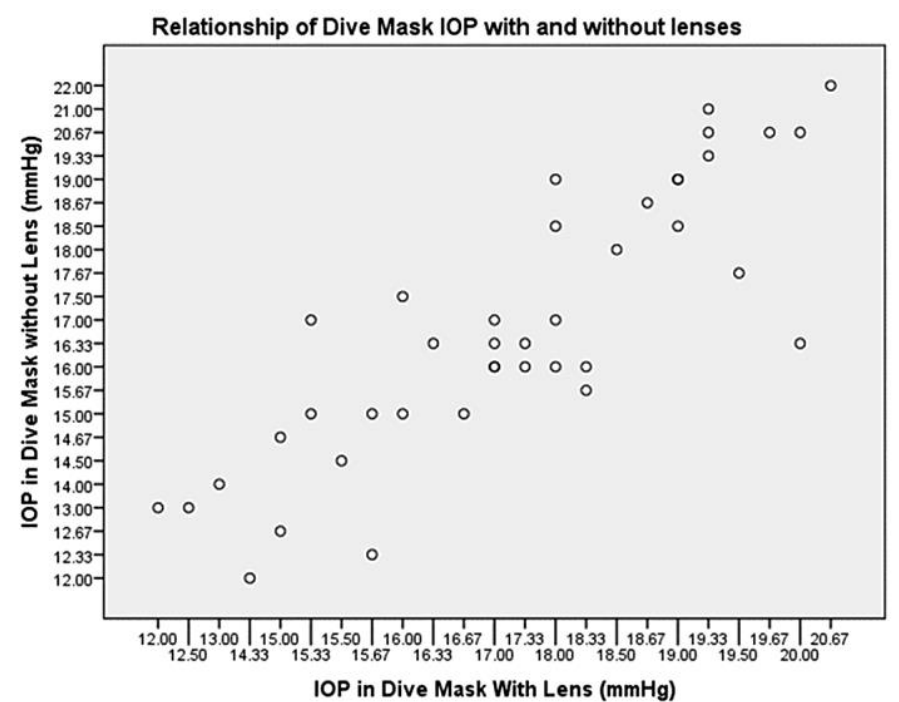

Fig. 2. Scatter plot illustrating the IOP measurements before and after donning of the diving mask. 